\title{
Promoção da fitoterapia no emprego de plantas medicinais com ação neurológica: uma revisão integrativa
}

Promotion of herbal medicine in the use of medicinal plants with neurological action: an integrative review

Promoción de la fitoterapia en el uso de plantas medicinales con acción neurológica: una revisión integradora

\section{Resumo}

Esta pesquisa teve como objetivo explanar que tipos de protocolos são aplicados para a utilização de plantas medicinais na atuação do sistema nervo, além de analisar a eficácia e segurança das plantas medicinais que agem no sistema neurológico. Para tanto, foram utilizadas as bases de dados eletrônicos MEDLINE/PUBMED, LILACS e SCIELO, que após critérios de exclusão e inclusão obteve-se x estudos. Os resultados mostraram que: Cymbopogon citratus e Melissa officinais apresentaram efeito sedativo e calmante, contra insônia e nervosismo, Curcuma longa L mostra potencial terapêutico no tratamento diversas doenças, inclusive no sistema nervoso central, Passiflora incarnara L. indicaram potencial efeito ansiolítico e sedativo, Lavandula officinalis é utilizado como óleo sendo essencial para uso através da aromoterapia, mostrando efeito calmante sedativo, a Petiveria alliace L. demostram desempenho sobre o sistema nervoso central. Conclui-se que apesar do potencial terapêutico das plantas medicinais frente aos distúrbios do sistema nervoso central, foram encontrados poucos estudos atualizados que avaliam a ação farmacológica dessas plantas medicinais. Vale ressaltar que esse estudo promove uma correlação entre o conhecimento popular e as diversas formas de aplicação do conhecimento científico dessas plantas medicinais.

Palavras-chave: Plantas medicinais; Fitoterapia; Sistema nervoso.

\begin{abstract}
This research aimed to explain what types of protocols are applied to the use of medicinal plants in the performance of the nervous system, in addition to analyzing the effectiveness and safety of medicinal plants that act on the neurological system. For that, the electronic databases MEDLINE/PUBMED, LILACS and SCIELO were used, which after exclusion and inclusion criteria were obtained x studies. The results showed that: Cymbopogon citratus and Melissa officinais had sedative and calming effects against insomnia and nervousness. Curcuma longa L shows therapeutic potential in the treatment of several diseases, including the central nervous system, Passiflora incarnara L. indicated a potential anxiolytic and sedative effect, Lavandula officinalis is used as an essential oil for use through aromatherapy, showing a calming sedative effect, Petiveria alliace L. demonstrates performance on the central nervous system. It is concluded that despite the therapeutic potential of medicinal plants against central nervous system disorders, few updated studies were found that assess the pharmacological action of these medicinal plants. It is noteworthy that this study promotes a correlation between popular knowledge and the various forms of application of scientific knowledge of these medicinal plants.
\end{abstract}

Keywords: medicinal plants; Phytotherapy; Nervous system.

\section{Resumen}

Esta investigación tuvo como objetivo explicar qué tipos de protocolos se aplican al uso de plantas medicinales en el desempeño del sistema nervioso, además de analizar la efectividad y seguridad de las plantas medicinales que actúan sobre el sistema neurológico. Para ello se utilizaron las bases de datos electrónicas MEDLINE / PUBMED, LILACS y 
SCIELO, que luego de criterios de exclusión e inclusión se obtuvieron x estudios. Los resultados mostraron que: Cymbopogon citratus y Melissa officinais tenían efectos sedantes y calmantes contra el insomnio y el nerviosismo, Curcuma longa $L$ muestra potencial terapéutico en el tratamiento de varias enfermedades, incluido el sistema nervioso central, Passiflora incarnara L.indicó un potencial efecto ansiolítico y sedante , Lavandula officinalis se utiliza como aceite esencial para uso a través de aromaterapia, mostrando un efecto sedante calmante, Petiveria alliace L. demuestra desempeño sobre el sistema nervioso central. Se concluye que a pesar del potencial terapéutico de las plantas medicinales frente a los trastornos del sistema nervioso central, se encontraron pocos estudios actualizados que evalúen la acción farmacológica de estas plantas medicinales. Es de destacar que este estudio promueve una correlación entre el conocimiento popular y las diversas formas de aplicación del conocimiento científico de estas plantas medicinales.

Palabras clave: Plantas medicinales; Fitoterapia; Sistema nervioso.

\section{Introdução}

As plantas medicinais, são elas classificadas como naturais ou cultivadas, tem sido utilizada para fins terapêuticos há milhares de anos por populações de vários países, a partir de então adquiriu-se a chamada sabedoria empírica passada de geração em geração, sendo uma das formas mais antigas que a população de várias regiões e culturas no mundo utilizam como método paliativo e com fins medicinais para tratamento de diversas doenças. Em função dos seus benefícios, podem ser apresentadas de três maneiras: substâncias in natura, manipuladas ou industrializadas (Almeida, 2013; Menezes et al., 2012).

Estudos farmacológicos indicam compostos que apresentam potencial terapêutico e de ações farmacológicas tais como propriedades ansiolíticas, sedativas e hipnóticas, tais como flavonoides (C-glicosídeos, possuindo efeito sedativo hipnótico, ansiolítico e analgésico), glicosídeos esteroidais e triterpenoides (glucoglispiranosil-estigmasterol, glucopiranosilácido oleanólico), saponinas (ginsenosideos, apresentaram atividade neuroprotetora sobre diferentes tipos de células neuronais), alcaloides (harmana, harmol, passiflorina ,responsáveis por produzir ações depressoras inespecíficas no SNC, promovendo efeito sedativo e tranquilizante), óleo essencial (citral, geraniol, cânfora, terpineóis, linalol, calmante, tranquilizante e insônia). Devido aos baixos níveis de toxicidades e de efeitos colaterais, quando comparados aos medicamentos sintéticos, se mostraram uma boa opção terapêutica, visto que estes exercem atividades mediadas pelo Sistema Nervoso Central (SNC), induzindo o indivíduo ao relaxamento e ao sono, já que suas ações sobre os neurotransmissores inibitórios que atuam no SNC, acalmam condições nervosas (Jager \& Saaby, 2011).

As diferentes misturas de fitocomplexos presentes nas plantas medicinais podem inibir a transmissão excitatória, promovendo a diminuição do estado de ansiedade, esse efeito sobre o SNC pode estar relacionado a afinidade desses compostos naturais com os sítios de ligação dos Benzodiazepínicos nos receptores Ácido Gama-Aminobutírico tipo A $\left(\mathrm{GABA}_{\mathrm{A}}\right)$, sendo esses os mais importantes receptores inibitórios no SNC, exibindo assim propriedades ansiolíticas (Hacke et al., 2020).

A fitoterapia é uma alternativa promissora, segura e eficaz, sendo usada uma variedade grande de plantas com esse propósito, onde já passaram por testes pré-clínicos, e devem passar por testes clínicos para atestar sua segurança e eficácia. A maioria das ervas medicinais testadas podem servir como alternativas aos ansiolíticos tradicionais em pacientes que não conseguem adesão à terapia convencional devido ao perfil de efeitos adversos que estes apresentam (Nóbrega et al., 2017).

Com base na utilização de plantas medicinais para fins terapêuticos, em 2006 foi executada no Brasil a Política Nacional de Práticas Integrativas e Complementares (PNPIC), e a Política Nacional de Plantas medicinais e fitoterápicos (PNPMF), com o objetivo de garantir à população acesso seguro e racional de plantas medicinais, utilizando a fitoterapia, promovendo assim o uso sustentável da biodiversidade no Sistema Único de Saúde (SUS), estabelecendo diretrizes que venham garantir o uso destes fitoterápicos (Caccia-Bava et al., 2017). 
Como forma de contribuir para a expansão de desenvolvimento, produção e dispensação dos produtos fitoterápicos, a Agência Nacional de Vigilância Sanitária (Anvisa) desenvolveu o Formulário de Fitoterápicos da Farmacopeia Brasileira, que já está na segunda edição, contendo 85 espécies (Agência Nacional de Vigilância Sanitária - Anvisa, 2021).

O Brasil possui uma das maiores diversidades vegetal do mundo, com isso a utilização das plantas medicinais poderia ser bastante benéfica para diminuição nos gastos com medicamentos, uma maior adesão ao tratamento e a contribuição para a validação cientifica sobre as espécies (Menezes, 2012). Considerando os promissores efeitos terapêuticos farmacológicos das plantas medicinais e a busca por tratamentos complementares, se faz necessário o entendimento dos protocolos e a efetividade da sua ação. Levando em consideração tudo que já foi exposto, este estudo tem como objetivo explanar que tipos de protocolos aplicados para autilização de plantas medicinais, além de analisar a eficácia e segurança das plantas medicinais que agem no SNC.

\section{Metodologia}

Este é um estudo de revisão de literatura bibliográfica, que busca é sintetizar o conteúdo de vários materiais e analisar criticamente as informações obtidas através de uma visão mais atual dos fatos relacionados. Três plataformas digitais foram utilizadas para a coleta de dados: Medical Literature Analysis and Retrieval System Online - MEDLINE/PUBMED, Literatura Latino-Americana e do Caribe em Ciências da Saúde - LILACS e Scientific Electronic Library Online - SciELO, com base em estudos publicados entre 2011 e 2021.

Foram adotados os seguintes critérios de exclusão: trabalhos que não corresponderam ao objetivo da pesquisa, não estavam disponibilizados na integra ou que estavam fora do período temporal demarcado. Os critérios de inclusão: caracterizamos artigos originais, completo e gratuito que tivessem foco no estudo de plantas medicinais e suas atividades farmacológicas sobre o sistema nervoso central, publicados nos idiomas inglês, espanhol e português.

Figura 1: Ilustração representativa do processo metodológico da revisão integrativa da literatura aplicada no estudo.

Fonte: Autores (2021). 
A pesquisa foi realizada no intervalo de tempo de agosto a outubro de 2021, com os seguintes descritores como fonte: "Plantas Medicinais", "Sistema Nervoso Central", "Plantas com Atividades Ansiolíticas" e "Propriedades Ansiolíticas de plantas medicinais". Os seguintes descritores foram usados na base de dados supracitadas e seguiram o fluxo metodológico representando na figura 1.

Em seguida, os resultados dos estudos foram sintetizados, procurando cobrir sua relevância e principais objetivos. Os dados dos estudos foram reunidos e sistematizados, caracterizando a análise crítica com o objetivo de gerar novos entendimentos sobre uma correlação entre a compreensão popular e as diversas formas de aplicação do conhecimento científico dessas plantas medicinais na forma de um quadro sinóptico.

\section{Resultados e Discussão}

Na triagem feita resultou em 35 artigos, os quais foram usadas para elaboração desde estudo, foram citadas 6 espécies de plantas medicinais que agem no SNC (quadro 1). Segundo Neto et al. (2015), plantas são fáceis de serem cultivadas em quintais e hortas, podendo ser preparados chás de diferentes formas, como através de infusão, decocção e maceração, sendo processos importantes para conservação adequada dos princípios ativos e consequentemente a sua eficácia terapêutica.

As plantas medicinais utilizadas pela população nas suas necessidades básicas de saúde devem ter eficiência terapêutica comprovada e complementada por estudos toxicológicos, envolvendo pesquisas com equipes multidisciplinares, como citam Lopes et al., (2013), a pesquisa científica valida saberes tradicionais e mantém vivo o saber popular, mas estudos farmacodinâmicos e toxicológicos são necessários para avaliar a dose, o risco e os benefícios do uso, garantindo a manutenção da saúde e segurança do usuário. (Fernandes; Félix; Nobre, 2016). O método a ser utilizado para a obtenção das preparações deve estar descrito na Farmacopeia Brasileira vigente ou, em sua ausência, nas Farmacopeias reconhecidas no país, conforme Resolução RDC n 37, de 6 de julho de 2009, e suas atualizações.

As plantas medicinais surgiram como alternativa natural de extrema importância para o sistema de saúde, e ainda para o desenvolvimento econômico, social e ambiental (Sen et al., 2011). Em 2015 uma lista de 60 espécies foi trabalhada por consultores da Organização Pan-Americana de Saúde (OPAS), no sentido da obtenção de informações cientificas e elaboração de novas monografas para a $2^{\circ}$ edição do formulário de fitoterápicos da farmacopeia Brasileira, aprovados pela Resolução da Diretoria Colegiada - RDC n ${ }^{\circ}$ 463, de 27 de janeiro de 2021, entre as quais foram inclusas no formulário de fitoterápicos da farmacopeia brasileira $2^{\circ}$ edição: Erva-cidreia (Melissa officinalis), Capim Santo (Cymbopogon citratus), Maracujá (Passiflora incarnata L.), Açafrão da terra (Cúrcuma L.) e Lavanda (Lavandula officinalis), onde tem se mostrado boas opções, apresentando efeitos adversos mínimos e relativa segurança de uso (Brasil, 2021).

Quando se refere as plantas medicinais, incluem-se as plantas medicinais descritas no Formulário de Fitoterápicos da Farmacopeia Brasileira (Brasil, 2018). O Quadro 1 foi construído a partir do objetivo da pesquisa, tendo em vista apontar novos entendimentos sobre uma correlação entre a compreensão popular e as diversas formas de aplicação do conhecimento científico dessas plantas medicinais. De acordo com a análise proposta, foram verificados os nomes populares, nomes científicos, indicações e interações de diferentes plantas medicinais que agem no SNC. 
Quadro 1: Relação do elenco de espécies selecionadas mediante ao estudo revisão bibliográfica.

\begin{tabular}{|c|c|c|c|c|c|}
\hline $\begin{array}{c}\text { NOME } \\
\text { CIENTIFICO/ } \\
\text { NOMES } \\
\text { POPULARES } \\
\end{array}$ & $\begin{array}{c}\text { PARTES } \\
\text { UTILIZADAS }\end{array}$ & $\begin{array}{l}\text { FORMA DE } \\
\text { USO }\end{array}$ & $\begin{array}{c}\text { INDICAÇÃO } \\
\text { TERAPÊUTICA }\end{array}$ & INTERAÇÕES & REFERÊNCIAS \\
\hline $\begin{array}{l}\text { Cymbopogon citratus/ } \\
\text { Capim- santo }\end{array}$ & Folhas / flores & Infusão & $\begin{array}{c}\text { Antiespasmódica; } \\
\text { Antimicrobiana; } \\
\text { Analgésica; } \\
\text { Calmante; } \\
\text { Diurético } \\
\end{array}$ & $\begin{array}{c}\text { Potencializar o efeito } \\
\text { de medicamentos } \\
\text { sedativos (calmantes) }\end{array}$ & $\begin{array}{l}\text { Lucena et al., } 2015 \\
\text { Peixoto et al., } 2015 \\
\text { Lima et al., } 2013\end{array}$ \\
\hline $\begin{array}{c}\text { Cúrcuma longa } L / \\
\text { Açafrão da terra } \\
\text { Gengibre amarela }\end{array}$ & $\begin{array}{l}\text { Raízes / } \\
\text { Rizomas }\end{array}$ & $\begin{array}{c}\text { Infusão } \\
\text { Tintura } \\
\text { Decocção } \\
\text { Capsulas: pó }\end{array}$ & $\begin{array}{c}\text { Antiviral, } \\
\text { Anti-inflamatória; } \\
\text { Antimicrobiana; } \\
\text { Hipoglicemiante; } \\
\text { Antioxidante; } \\
\text { Antidepressiva } \\
\text { Antiespasmódica, } \\
\text { Anticonvulsivante } \\
\text { Neuroprotetor } \\
\end{array}$ & $\begin{array}{c}\text { Interações com } \\
\text { anticoagulantes, } \\
\text { aumenta o risco a } \\
\text { hemorragias. } \\
\text { O consumo associado } \\
\text { ao paracetamol pode } \\
\text { aumentar a toxicidade } \\
\text { deste devido à } \\
\text { indução de CYP1A2 }\end{array}$ & $\begin{array}{c}\text { Muniz et al., } 2013 \\
\text { Alonso, } 2016 \\
\text { Wang,Q 2016 } \\
\text { Kim; Kim; Yang, } 2014 \\
\text { Grandi, 2014 } \\
\text { Pereira et al., } 2014 \\
\text { Kuptniratsaikul et al., } \\
\text { 2014 } \\
\text { Mazzari \& Prieto, } 2014 \\
\end{array}$ \\
\hline $\begin{array}{c}\text { Lavandula } \\
\text { angustifólia/ } \\
\text { Lavanda/alfazema }\end{array}$ & Folhas / flores & $\begin{array}{c}\text { Infusão } \\
\text { Tintura } \\
\text { Destilação das } \\
\text { flores }\end{array}$ & $\begin{array}{c}\text { Antibacteriana } \\
\text { Antioxidante; } \\
\text { Antiespasmódica; } \\
\text { Anticonvulsivante; } \\
\text { Antifúngica; } \\
\text { Anti-inflamatória. } \\
\text { Analgésica } \\
\text { Calmante } \\
\text { Sedativa; } \\
\end{array}$ & $\begin{array}{l}\text { Não deve ser utilizado } \\
\text { concomitantemente } \\
\text { com depressores do } \\
\text { Sistema Nervoso } \\
\text { Central (SNC), como } \\
\text { álcool etílico, } \\
\text { benzodiazepínicos e } \\
\text { narcóticos. }\end{array}$ & $\begin{array}{c}\text { Bagheri-Nesami, } 2017 \\
\text { Lyu et al., } 2018 \\
\text { Andrioli, B.V,2014 }\end{array}$ \\
\hline $\begin{array}{c}\text { Melissa officinalis/ } \\
\text { Erva-cidreira/ } \\
\text { Capim-limão }\end{array}$ & Folhas /flores & $\begin{array}{c}\text { Infusão } \\
\text { Tintura } \\
\text { Hidrodestilação } \\
\text { Extrato seco } \\
\text { Extrato fluido }\end{array}$ & $\begin{array}{c}\text { Anti-inflamatório; } \\
\text { Antiespasmódica } \\
\text { Antioxidante } \\
\text { Antifúngica, } \\
\text { Ansiolítica } \\
\text { Bactericida } \\
\text { Sedativa }\end{array}$ & $\begin{array}{c}\text { Potencializar os } \\
\text { efeitos hormonais em } \\
\text { pessoas com } \\
\text { hipotireoidismo } \\
\text {. }\end{array}$ & $\begin{array}{c}\text { Barnes, J, } 2012 \\
\text { Brasil, 2011; } 2021 \\
\text { Ema, 2013 } \\
\text { Pereira et al., } 2014 \\
\text { Colussi et al., } 2011\end{array}$ \\
\hline $\begin{array}{c}\text { Passiflora incarnata } \\
\text { Flor de paixão / } \\
\text { Maracujá }\end{array}$ & $\begin{array}{l}\text { Partes aéreas } \\
\text { Folhas/caules }\end{array}$ & $\begin{array}{l}\text { Infusão/tintura } \\
\text { extrato fluido }\end{array}$ & $\begin{array}{c}\text { Ansiolítico } \\
\text { Analgésico } \\
\text { Antiespasmódicos } \\
\text { sedativo }\end{array}$ & $\begin{array}{c}\text { Não usar em casos de } \\
\text { tratamento com } \\
\text { sedativos e } \\
\text { depressores do } \\
\text { sistema nervoso. }\end{array}$ & $\begin{array}{c}\text { Miroddi et al., } 2013 \\
\text { Barnes et al., } 2012 \\
\text { Ema, } 2014\end{array}$ \\
\hline $\begin{array}{c}\text { Petiveira Alliace } \\
\text { guiné /amansa senhor }\end{array}$ & $\begin{array}{c}\text { Folha e haste } \\
\text { /Raiz/flores }\end{array}$ & $\begin{array}{c}\text { Infusões } \\
\text { Decocção } \\
\text { Banhos } \\
\text { Extratos seco }\end{array}$ & $\begin{array}{l}\text { Antiespasmódicas, } \\
\text { abortivas, } \\
\text { anti-inflamatório, } \\
\text { anticancerígeno } \\
\text { estimulantes } \\
\text { antifúngica } \\
\text { laxativas, } \\
\text { sedativo, }\end{array}$ & $\begin{array}{l}\text { Tomar mediante a } \\
\text { prescrição médica; } \\
\text { alta ingestão pode } \\
\text { resultar em; } \\
\text { intoxicação, insônia e } \\
\text { aborto. }\end{array}$ & $\begin{array}{c}\text { Fletes-Arjona et al., } 2013 \\
\text { Kim, Kubec \&Musah, } \\
2017 \\
\text { Lavezo et al., } 2015 \\
\text { Gao et al., 2015 } \\
\text { Garrett et al., 2012 } \\
\text { Feng et al., } \\
2014 \\
\text { Wang et al., } 2016\end{array}$ \\
\hline
\end{tabular}

Fonte: Autores (2021).

Segundo Avoseh (2015) Cymbopogon citratus (D.C.) é uma espécie exótica herbácea pertencente à família Graminacea, de origem Índiana, sendo conhecido popularmente como capim-limão, capim-santo, capim-cidreira e encontra-se difundida em vários países e aclimatada nas regiões tropicais do Brasil, com longas folhas aromáticas, estreitas, agudas e ásperas e com nervura central proeminente. É muito utilizada na forma de chás, tanto com as folhas fervidas em água, como em infusão. É extraído da planta o óleo essencial que tem como composição o limoneno, citronelal, mirceno, geraniol, e seu principal o citral. Estudos tem mostrado que o capim santo, muito utilizado pela população brasileira empiricamente, tem efeitos antibacterianos e calmantes, comprovados cientificamente (Peixoto et al., 2015; Lucena et al., 2015).

A Curcuma longa L. é uma planta pertencente à família das Zingiberaceae (Grandi, 2014). Popularmente conhecida como açafrão da Índia, açafrão, açafroa, açafrão-da-terra, cúrcuma, gengibre dourado e mangarataia muito usado na culinária 
(Barnes; Anderson; Phillipson,2012). É utilizada como tempero, especialmente na região continental da Índia. Sendo consumido principalmente na forma de pó seco, utilizado para coloração alimentícia devido à sua forte coloração amarela, associada aos efeitos terapêuticos e dietéticos, ganhou espaço por suas propriedades etnomedicinais que incluem: atividade hepática, gastroprotetora, anti-inflamatória, antimicrobiana, anti-HIV, hipoglicemiante, hipoglicemiante, antiagregante, dermatológica, oftalmológica, antioxidante, atuam no sistema respiratório, sistema reprodutor, no sistema digestório e no SNC (Alonso, 2016).

Segundo Muniz et al., (2013) ação neuroprotetora da Curcuma longa L. está relacionada aos compostos da curcumina, suprimindo os danos oxidativos, inibe peroxidases responsáveis por grande parte das cito patologias do Alzheimer.

Entretanto, Chin et al., (2013) aponta que a mesma apresenta baixa biodisponibilidade, pouca solubilidade em água e estabilidade em solução, além de rápida passagem pelo intestino e metabolização hepática, fatos que levam a necessidade de novas pesquisas, elaboração de métodos, a fim de elucidar as lacunas do uso da curcumina em doenças neurodegenerativas.

Algumas plantas, incluindo a Cúrcuma longa, embora encontrada na literatura a atividade farmacológica de indutores do sono e ansiolítica, esses dados ainda não estão apresente no Formulário de Fitoterápicos da Farmacopeia Brasileira (Brasil, 2011; 2021) e nem no Memento Fitoterápico (Brasil, 2016). É importante ressaltar que estas duas referências são oficiais, produzidas pela Anvisa. Acredita-se que pelo uso não muito difundido em todo território nacional e por escassas informações de segurança e eficácia, esses dados não estão ainda disponíveis, considerando que há um setor específico dentro da Anvisa que atualiza periodicamente as informações de pesquisas baseadas em evidência, padronizando a informação para o uso racional de fitoterápicos e plantas medicinais. Portanto, recomenda-se cautela quanto ao uso dessas plantas até que sejam apresentados mais dados de eficácia e segurança.

Já a Lavandula angustifólia é um membro da família da Lamiaceae e do gênero Lavandula, é nativa da região do mediterrâneo, sendo cultivada para fins industriais principalmente no Sul da Europa, mas seu cultivo se disseminou internacionalmente, conhecida popularmente como lavanda ou alfazema, que contém os princípios ativos como acetato de linalil, cariofileno, taninos, saponina ácida, princípio amargo e os álcoois geraniol, furfurol, linalol e seus ésteres (Machado, 2013).

Bagheri-Nesami, (2017) mostra em seus estudos que o Linalol e acetato de linalila são os componentes principais, esses compostos têm propriedades analgésicas e sedativas, estimulam o Sistema Parassimpático que leva à diminuição da frequência cardíaca, consequentemente melhorando a função cardíaca.

A lavandula angustifolia aumenta o efeito do ácido gama-aminobutírico (GABA) na amígdala e tem efeitos narcóticos e sedativos semelhantes aos dos Benzodiazepínicos. Além de suas características antibacterianas, antifúngicas e carminativas, que aumentam a cicatrização de ferida desintoxicação de enzimas associadas a picadas de insetos, a alfazema também é usada para relaxamento espiritual, para fins terapêuticos e para a regulação de distúrbios do sono (Lyu et al., 2018).

A aromaterapia consiste na aplicação terapêutica de óleos essenciais que são substâncias empregadas com a finalidade de equilibrar as emoções, melhorar o bem-estar físico e mental, e que atuam de diversas formas no organismo, podendo ser absorvidas por meio da inalação pelas vias aéreas, por uso tópico ou ingestão desencadeando uma sequência de reações químicas no organismo, capaz de reestabelecer o equilíbrio, reduzindo os sintomas e tratando as doenças (Gnatta; Dornellas; Silva, 2011).

A lavanda foi inserida no Formulário de Fitoterápicos em 2021, sendo indicada no alívio da ansiedade e insônias leves. O uso contínuo não deve ultrapassar 15 a 20 dias, podendo ser repetido o tratamento se for necessário após 7 dias de intervalo. Porém relata-se cautela pois como efeitos adversos pode desencadear sonolência, cefaleia, constipação intestinal, dermatite de contato, confusão mental e hematúria em doses elevadas ou tóxicas, ou em pessoas hipersensíveis (Pereira et al., 2019). 
De acordo com pesquisas de Rasmussen (2011), Melissa officinalis pertence à família Lamiaceae, uma planta aromática nativa da Europa. Devido ao seu largo uso como planta aromática e medicinal é amplamente cultivada em hortas domésticas no sul do Brasil, conhecida popularmente como melissa e erva cidreira. Essa planta também citada no Formulário de Fitoterápicos da Farmacopeia Brasileira é indicada como antiespasmódico, sedativo leve e ansiolítico, (Nasri H,2013). As suas folhas e inflorescências são usadas na medicina tradicional por seus efeitos nas funções do SNC, empregadas na forma de chá como calmantes, possui efeito que pode modular várias medidas de comportamento, como um moderado sedativo em transtorno do sono, na atenuação de sintomas de desordens nervosas, inclusive a redução de excitabilidade, ansiedade e tensão, papel importante no controle das emoções, demonstrou também ser um bom agente antibacteriano e antifúngico , antioxidante, anti-inflamatório (Shafiei, 2012).

Análises da constituição química dos extratos de parte aéreas de M. officinalis L. evidenciaram a presença de componentes como taninos, derivados dos ácidos rosmarínicos e caféico, ácidos triterpenóides e flavanóides. Entretanto, os componentes majoritários presentes na folha são: Citronelal (2-40\%), citral (10-30\%), seguido pelo $\beta$-cariofileno, germancreno D, ocimeno e citronelol (Colussi et al., 2011; Brasil, 2016). Deve-se observar que existem diversas outras plantas utilizadas pela comunidade como nome popular de "cidreira" ou "melissa", o que pode causar confusão em relação a sua identificação botânica.

A Passiflora incarnata L. pertencem à família Passifloraceae que possui diversas espécies estudadas dentre as quais se destacaram Passiflora incarnata, Passiflora alata Dryand (Maracujá-doce), e Passiflora edulis Sims (maracujá azedo), que são amplamente utilizadas na medicina tradicional em todos os países, conhecido popularmente como maracujá-vermelho, flor-da paixão e maracujá, originária da América do Norte, é uma das plantas medicinais mais importantes (Ema, 2014).

É muito utilizada no tratamento da ansiedade, por ter ação ansiolítica e sedativa agindo como um depressor inespecífico do SNC (Miroddi et al., 2013). Em seus constituintes é possível encontrar alcalóides, flavonóides, glicosídeos cianogênicos, fração de esteroides e saponinas. Os flavonoides encontrados são principalmente do tipo $C$ - glicosídeos, possuindo efeito sedativo, hipnótico, ansiolítico e analgésico (Gosmann et al., 2011; Rodrigues et al., 2015).

O farmacógeno da $P$. incarnata é encontrado nas partes aéreas da planta constituídas por folhas e caules, que são as partes de interesse para a produção de fitoterápicos apresentados na forma de comprimidos e cápsulas contendo o extrato seco da passiflora (Brasil, 2016). Os efeitos farmacológicos são mediados via modulação do sistema GABA, incluindo afinidades aos receptores $\mathrm{GABA}_{\mathrm{A}}$ e $\mathrm{GABA}_{\mathrm{B}}$, e sobre a receptação de GABA e seu mecanismo de ação envolve a potenciação do sistema GABAérgico (Appel et al., 2011).

Estudos fitoquímicos têm contribuído para a descoberta de grande variedade de substâncias biologicamente ativas produzidas em diferentes partes da planta. E dentre esses estudos fito químicos a espécie apresenta características medicinais sedativas. Por esta razão, Andrade et al., (2012) realizaram um screening comportamental, a fim investigar a ocorrência de tais atividades a partir da administração do extrato de partes totais de P. alliacea. Um dos efeitos observados, está relacionado a melhora na memória de longa duração (MLD), induzida pelo pré-tratamento com o extrato de planta total na dose de 900 mg/Kg. No entanto, Andrade et al., (2012) não observou atividade (melhora ou prejuízo) sobre a memória de curta duração (MCD) e aprendizagem. Extrato feito das partes da raiz mostrou um efeito ansiolítico e é usada na religião afro-brasileira para criar "equilíbrio e harmonia no meio ambiente" e "para acalmar" (Garcia et al., 2016).

Há relatos na literatura que os níveis de toxidade da raiz dessa planta dependem da dose e frequência de uso, estes extratos podem vir apresentar atividade citotóxica, recomenda-se seu uso sobre orientação médica. Sendo algumas recomendações citadas por Nasri e Shirzad (2013), as quais deveriam ser consideradas ou informadas aos usuários de plantas com fins medicinais: utilizar somente plantas referenciadas em publicações, evitar aquelas novas ou com ação não comprovada, descontinuar o uso se não for obtido benefício ou resultado ou se houver reações adversas, não aderir ao uso de 
quaisquer produtos sem conhecimento, considerar as interações medicamentosas e as contraindicações individuais e evitar plantas medicinais durante a gravidez. Os autores enfatizam a gravidez, considerando o risco de teratogenicidade e outros, pois muitas plantas não têm esse risco determinado.

\section{Considerações Finais}

A pesquisa permitiu verificar a importância da inserção das plantas medicinais no cuidado a saúde e seu grande potencial de utilização, no qual são usadas há muito tempo por nossos antepassados e são conhecidas por terem um papel importante na cura e tratamento de algumas doenças. Em algumas comunidades, essas plantas simbolizam a única forma de tratamento de determinadas patologias. Estima-se que $65 \%$ da população incorporam o uso de plantas da medicina tradicional aos cuidados médicos. Contudo, as estimativas revelam que do total de aproximadamente 250.000 espécies de plantas existentes no globo terrestre apenas $10 \%$ foram testadas em ensaios biológicos. Ainda assim, estima-se que $40 \%$ dos medicamentos atualmente disponíveis foram desenvolvidos a partir de fontes naturais e cerca de $60 \%$ dos fármacos aprovados no período entre 1981 e 2002, eram produtos naturais ou foram desenvolvidos a partir destes.

Além disso, a maioria das ervas medicinais devidamente testadas podem servir como alternativas para pacientes que não conseguem adesão a terapia convencional, devido ao perfil de efeitos adversos que estes apresentam. Diante disso, para a avaliação do potencial enquanto planta medicinal faz-se necessários estudos de validação farmacológica para o emprego clínico objetivando benefícios à saúde humana.

Destaca-se que pouco se sabe sobre os possíveis mecanismos pelos quais a planta exerce suas atividades, o que indica a necessidade de dar prosseguimentos as pesquisas, para plena compreensão de seu potencial farmacológico. Sugere-se ainda a realização de novas pesquisas que abordem o uso clínicos das plantas medicinais, a fim de embasar seu uso na prática clínica.

\section{Referências}

Andriolli, B. V. (2014). Micropropagação de Lavanda (Lavandula x intermedia Emeric ex Loisel., Lamiceae) variedade Grosso para extração de óleo essencial.Trabalho de Conclusão de Curso (Graduação) - Curso em Ciências Rurais, Universidade Federal de Santa Catarina.10(8), 24.

Avoseh, O., Oyedeji, O., Rungqu, P., Nkeh-chungag, B., \& Oyedeji, A. (2015). Cymbopogon Species, Ethnopharmacology, Phytochemistry and the Pharmacological Importance, Molecules, 20(5), 7438-53. https://doi.org/10.3390/molecules20057438.

Appel, K., Rose, T: Fiebich, B., Kammler, T., Hoffmann, C., \& Weiss, G. (2011). Modulation of the $\gamma$-aminobutyric acid (GABA) system by Passiflora incarnata L. Phytotherapy research: PTR, 25(6), 838-843. https://doi.org/10.1002/ptr.3352

Andrade, T. M., Melo, a.s, Dias, R. G. C., Varela, E. L. P., Oliveira, F. R., Vieira, J. L. F., Andrade, M. A., Baetas, A. C., Monteiro, M. C., \& Maia, C. S. F. (2012). Potential behavioral and pro-oxidant effects of Petiveria alliacea L.extract in adult rats. Journal of Ethnopharmacology, 143(2,28), 604-610.

Almeida \& Ana Beatriz Albino et al. (2013). Anti-inflammatory intestinal activity of Arctium lappa L. (Asteraceae) in TNBS colitis model. Journal of Ethnopharmacology, 146(1), 300-310. http://hdl.handle.net/11449/74805.

Alonso, J. (2016). Tratado de Fitofarmácos e $\quad$ Nutracêuticos. $\quad$ São $\quad$ Paulo: $\quad A \quad C \quad$ Farmacêutica, 20 , (3),364 - 373. https://doi.org/10.25110/arqsaude.v20i3.2016.5871

Anvisa. Agencia Nacional de Vigilancia Sanitaria, (2021). Formulário de Fitoterápicos, (2a ed.).

Barnes, J., Anderson, L. A., \& Phillipson, J. D. (2012). Fitoterápicos. (3a ed.), Artmed, 720.

Bagheri-Nesami M, Shorofi S A, Nikkhah A, \& Espahbodi f. (2017). The effects of lavender essential oil aromatherapy on anxiety and depression in haemodialysis patients,3(1),8-13.

Brasil, Agência Nacional de Vigilância Sanitária (2011). Formulário de Fitoterápicos da Farmacopéia Brasileira. Anvisa, p. 126.

Brasil, Ministério da Saúde. Agência Nacional de Vigilância Sanitária. Resolução RDC N 84, de 17 de junho de 2016 . Dispõe sobre a aprovação do Memento da Farmacopeia Brasileira, $1^{\text {a }}$ edição, p.115.

Brasil, Ministério da Saúde. Agência Nacional de Vigilância Sanitária (2021). Resolução RDC N 463, de 27 de janeiro de 2021 . Dispõe sobre a aprovação do Formulário de Fitoterápicos da Farmacopeia Brasileira, 2ª edição, p. 223.

Brasil, Ministério da Saúde. Agência Nacional de Vigilância Sanitária (2018). Resolução RDC N 225 , de 11 de abril de 2018 . Dispõe sobre a aprovação do $1^{\circ}$ Suplemento do Formulário de Fitoterápicos da Farmacopeia Brasileira, 1ª edição. 
Caccia-Bava., M. do C., Gullaci, G., et al. (2017). Disponibilidade de medicamentos fitoterápicos e plantas medicinais nas unidades de atenção básica do Estado de São Paulo: resultados do Programa Nacional de Melhoria do Acesso e da Qualidade da Atenção Básica (PMAQ). Ciência \& Saúde Coletiva. 22( 5), 1651-1659.

Chin, D. et al. (2013) Neuroprotective Properties of Curcumin -Merits and Limitations. Current Medicinal Chemistry 20(32). 189-194.

Colussi, T. C., Dalmolin, L. F., Pachtmann, M., \& Freitas, G. B. L. (2011). Melissa officinalis L.: Características gerais e biossíntese dos principais metabólitos secundários. Revista de Biologia e Farmácia. 18 (4), 89-100. http://dx.doi.org/10.5380/acd.v18i4.56123

EMA, European Medicines Agency. (2013). Community herbal monograph on Melissa officinalis L., folium. London:Committee on Herbal Medicinal Products (HMPC). (2a ed.), p.128.

EMA, European Medicines Agency. (2014). Community herbal monograph on Passiflora incarnata L., herba. London:Committee on Herbal Medicinal Products (HMPC). (2a ed.), p.145.

Fernandes, C. O. M., Félix, S., \& Nobre, M. O. (2016). Toxidade dos fitoterápicos de interesse do sus: uma revisão. Semina: Ciências Biológicas e da Saúde, 37(1).91. http://dx.doi.orq/10.5433/1679-0367.2016v37n1p91

Fletes-Arjona V. M., Soto-Dominguez A., Garcia-Garza R., Moran-Martinez J., Benitez-Valle C., Castaneda-Martinez A., Montalvo-Gonzalez R., \& BecerraVerdin. (2013) Em Alterações morfológicas no trato respiratório de ratos wistar induzidas por vapores da raiz de Hierba del Zorrillo (Petiveria alliacea) do sudoeste do México. Int J Morphol, 12(6), 121-127. https://doi.org/10.4162/nrp.2018.12.6.503

Feng, Z., Hao, W., Lin, X., Fan, D, \& Zhou, J. (2014). A atividade antitumoral de flavonóides totais de Tetrastigma hemsleyanum Diels et Gilg está associada à inibição de células T reguladoras em camundongos. OncoTargets and therapy, (7), 947-956.

Gnatta, J. R.., Dornellas., E. V. \& Silva., M. J. P. (2011). O uso da aromaterapia no alívo da ansiedade. Acta Paulista de Enfermagem, 24 ( 2), $257-263$.

Grandi, T. S. M. (2014). Tratado das plantas medicinais Mineiras, Nativas e Cultivadas. Adaequatio Estúdio. P.1204.

Gao, Y., Zhang, M., Wu, T., Xu, M., Cai, H., \& Zhang, Z (2015). Effects of D-Pinitol onInsulin Resistance through the PI3K/Akt Signaling Pathway in Type 2 DiabetesMellitus Rats. Journal of Agricultural and Food Chemistry. 63(26), 6019-6026.

Garrett \& Rafael et al. (2012). Antiherpetic activity of a flavonoid fraction from Ocotea notata leaves. Revista Brasileira de Farmacognosia. $22(2), 306-313$. Gosmann, G. et al. (2011). Composição química e aspectos farmacológicos de espécies de Passiflora L. (Passifloraceae). Revista Brasileira de Biociências. 9(1),88-89. http://www.ufrgs.br/seerbio/ojs/index.php/rbb/article/view/1607.

Garcia, D., Ribeiro, C., Santos, J. F. L., Soares, J. A. R., Antonio, R. L., Santos, T. S. D. dos, \& Rodrigues, E. (2016). Defumadores com possível efeito ansiolítico utilizados no centro de Umbanda Caboclo Ubirajara e Exú Ventania, Diadema, SP: Um Estudo Etnofarmacológico. Ethnoscientia. 1(1), 1-12.

Hacke, A. C. M. et al. (2020). Anxiolytic properties of Cymbopogon Citratus (DC.) stapf extract, essential oil and its constituents in zebrafish (Danio rerio). Journal Of Ethnopharmacology, 260(5), 13036. https://doi.org/10.1016/j.jep.2020.113036.

Jager, A. K., \& Saaby, L. (2011). Flavonóides e o SNC. Molecules ,16(2), 1471-485. https://doi.org/10.1590/S1516-05722013000200008

Kim, S., Kubec, R., Musah., \& Rabi A. (2017) Antibacterial and antifungal activity of sulfur-containing compounds from Petiveria alliacea L. Journal Of Ethnopharmacology, Oxford, 1-2(104), 188-192. https://doi.org/10.31692/2526-7701.IVCOINTERPDVAgro.2019.0066.

Kuptniratsaikul, V., Dajpratham, P., Taechaarpornkul, W., Buntragulpoontawee, M., Lukkanapichonchut, P., Chootip, C., Saengsuwan, J., Tantayakom, K., \& Laongpech, S. (2014). Efficacy and safety of Curcuma domestica extracts compared with ibuprofen in patients with knee osteoarthritis: a multicenter study. Clinical Interventions in Aging. (9), 451-458. https://www.ncbi.nlm.nih.gov/pmc/articles/pmc3964021

Kim, M. H., Kim, S. H., \& Yang, W. M. (2014). Mechanisms ofaction of phytochemicals from medicinal herbs in the treatment of Alzheimer's disease. Planta Med, 80(15), 1249-58.

Lucena, B. F. F. et al. (2015). Avaliação da atividade antibacteriana e moduladora deaminoglicosídeos do óleo essencial de Cymbopogon citratus (DC.) Stapf. Acta biol. Colomb. 20(01), 39-45. http://dx.doi.org/10.15446/abc.v20n1.41673

Luz, D. A., Pinheiro., A. M, Silva, M. L, Monteiro., M. C, Prediger., R. D, Maia., C. S. F., \& Fontes-júnior, E. A. (2016). Etnobotânica, fitoquímica e efeitos neurofarmacológicos de Petiveria alliacea L. (Phytolaccaceae): Uma revisão. J. Ethnopharmacol. (185), 182-201. https://doi.org/10.1016/j.jep.2016.02.053

Lavezo, A. et al. (2015) Estresse osmótico na germinação de sementes de Petiveria alliacea L. Revista Brasileira de Plantas Medicinais, 17 (4), $622-630$.

Lima, L., Polizelli, M., de Miranda, T., de Araújo, I., \& Pinto, D. (2013). a prática da fitoterapia a partir do conhecimento popular em três comunidades do valentina, joão pessoa - paraíba. Revista De Ciências Da Saúde Nova Esperança, 11(3), 23 - 34. https://doi.org/10.17695/revnevol11n3p23 - 34

Lyu, J. et al. (2018) The effects of music therapy on cognition, psychiatric symptoms, and activities of daily living in patients with Alzheime'r diseases. J. Alzheimer disease. 64 (4), 1347-1358.

Lopes Marielle, I. et al. (2013). Uso racional de plantas medicinais: um resgate popular na região do Vale do Assu-RN. Informativo Técnico do Semiárido, Pombal, 7(1): 23-29. https://doi.org/10.1590/S0103-73312021310218

Lucena, B. F. F. et al. (2015). Avaliação da atividade antibacteriana e moduladora deaminoglicosídeos do óleo essencial de Cymbopogon citratus (DC.) Stapf. Acta biol. Colomb. 20(01), 39-45. https://doi.org/10.1016/j.biopha.2017.01.158

Machado, M. P. et al. (2013). Propagação In vitro e caracterização química do óleo essencial de lavandula angustifólia cultivada no Sul do Brasil. Cien. Rural, 43(2), 283-289. 
Research, Society and Development, v. 10, n. 4, e393101422281, 2021

(CC BY 4.0) | ISSN 2525-3409 | DOI: http://dx.doi.org/10.33448/rsd-v10i14.22281

Menezes, V. A. et al. (2012) Terapêutica com plantas medicinais: percepção de profissionais da Estratégia de Saúde da Família de um município do agreste Pernambucano. Rev. Odonto (São Bernardo do Campo), 20(39), 111 - 122. https://core.ac.uk/download/pdf/229059055.pdf

Muniz, R., Cavalcante, A., Pereira, L, Sousa, F, Vasconcellos, S, Macedo, D, Viana, G, \& Fonteles, M. (2013). plantas medicinais da renisus de atuação central. Infarma - Ciências Farmacêuticas, 24(1-3), 75-80. http://www.revistas.cff.org.br/?journal=infarma\&page=article\&op=view\&path\%5B $\% 5 \mathrm{D}=361$

Mazzari, A. L. D. A, \& Prieto, J. M. (2014). Herbal medicines in Brazil: pharmacokinetic profile and potential herb-druginteractions. Frontiers in pharmacology. (5), 162. https://doi.org/10.3389/fphar.2014.00162

Miroddi, M, Calapai, G, Navarra, M., Minciullo, P. L, \& Gangemi, S., (2013). Passiflora incarnata L: etnofarmacologia, aplicação clínica, segurança e avaliação de ensaios clínicos. J. Ethnopharmacol, (150), 791-804.

Neto, I. R. S., AlveS, M. G. L, \& Martins, M. T. C. S. (2015). Utilização de plantas medicinais pelos grupos de idosos e de jovens no municipio de Parari PB. Revista Acadêmico científica, 07(1),1-15.

Nasri H., Rafieian-Kopaei M, (2013). Estresse oxidativo e prevenção do envelhecimento. Int J Prev Med. 2013 (4), 1101-1102.

Nóbrega, A. L., Ugulino, P. T. D., Cajá, D. F., Dantas, A. E. F. (2017). A importância da orientação dos profissionais das equipes de saúde da família acerca do uso da fitoterapia. Revista Brasileira de Educação e Saúde, 7 (1), 43-48. https://doi.org/10.33448/rsd-v9i11.10175

Nunes, M. G. S., Bernardino, A. O, \& Martins, R. D. (2015). Uso de plantas medicinais por pessoas com hipertensão. Revista Rene, 16 (6), 775-81.

Peixoto, M. I. et al. (2015). Plantas medicinais utilizadas por idosos da zona rural deFagundes - PB. Congresso internacional de envelhecimentohumano,02(01). http://www.editorarealize.com.br/editora/anais/cieh/2015/TRABALHO_EV040_MD4_SA3_ID337_27082015172304.pdf

Pereira, A. M. S., Bertoni, B. W, Silva, C. C. M, Ferro, D, Carmona, F, Cestari, I. M, Barbosa,M. G. H. (2014). Formulário Fitoterápico Farmácia da Natureza. (2a ed.), 407.

Pereira, M. C. L. et al. (2019). Use of medicinal plants in care of women with gynecological diseases: integrative review. International Journal of Development Research, 09(03), 26373- 26380.

Rodrigues da Silva, L. et al. (2015). Flavonoides: constituição química, ações medicinais e potencial tóxico. Acta toxicológica argentina, 23 (1), 36-43.

Rasmussen (2011) P. Erva-cidreira - Melissa officinalis, também conhecida como erva-cidreira, erva-cidreira, erva-cidreira, Melissa, melissengeist .J Prim Health Care, 22(3), 165-166. https://doi.org/10.1177 / 2156587216663433

Silva, G. K. F, (2015). Efetividade das medicinas tradicionais e complementares para os transtornos mentais comuns: uma revisão. Trabalho de Conclusão de Curso (Residência Multiprofissional em Saúde Coletiva) - Centro de Pesquisas Aggeu Magalhães, Recife p.35.

Sen S., Chakraborty R., \& Biplab B. (2011). Challenges and opportunities in the advancement of herbal medicine: India's position and role in a global context. JournalofHerbal Medicine, 1(1- 4), 67- 75. https://doi.org/10.1016/j.hermed.2011.11.001

Silva, L. E., Quadros, D. A., \& Maria Neto, A. J. (2015). Estudo etnobotânico e etnofarmacológico de plantas medicinais utilizadas na região de matinhos PR. Ciência e Natura, Santa Maria, 37(2), 266-276. http://dx.doi.org/10.5902/2179460X15473

Shafiei, Z. et al. (2012). Antibacterial Activity of Myristica fragrans against Oral Pathogens. Evidence Based Complementary and Alternative Medicine, 8(2) 135-152.

Wang, Q., Jin, J., Dai, N., Han, N., Han, J., \& Bao, B. (2016). Anti-inflammatory effects, nuclear magnetic resonance identification, and high-performance liquid chromatography isolation of the total flavonoids from Artemisia frigida. Journal of Food and Drug Analysis, 24(2), 385-391. 